Research Article

\title{
VIV Fracture Investigation into 3D Marine Riser with a Circumferential Outside Surface Crack
}

\author{
Jun Liu, ${ }^{1,2}$ Zhigang Du, ${ }^{2}$ Xiaoqiang Guo $\mathbb{D}^{1,2,3}$ Liming Dai, ${ }^{3}$ Liang Huang, ${ }^{4}$ and Xiao $\mathrm{Li}^{2}$ \\ ${ }^{1}$ School of Mechanical Engineering, Chengdu University, Chengdu 610106, China \\ ${ }^{2}$ School of Mechatronic Engineering, Southwest Petroleum University, Chengdu 610500, China \\ ${ }^{3}$ Industrial Systems Engineering, University of Regina, Regina SK S4S 0A2, Canada \\ ${ }^{4}$ Zhanjiang Branch, CNOOC (China) Co. Ltd., Zhanjiang 524057, China
}

Correspondence should be addressed to Xiaoqiang Guo; 202099010174@swpu.edu.cn

Received 7 July 2021; Accepted 28 October 2021; Published 12 November 2021

Academic Editor: Fabio Botta

Copyright $\odot 2021$ Jun Liu et al. This is an open access article distributed under the Creative Commons Attribution License, which permits unrestricted use, distribution, and reproduction in any medium, provided the original work is properly cited.

\begin{abstract}
Vortex-induced vibration (VIV) is one of the most common dynamic mechanisms that cause damage to marine risers. Hamilton's variational principle is used to establish a vortex-induced vibration (VIV) model of a flexible riser in which the wake oscillator model is used to simulate cross-flow (CF) and inline flow (IL) vortex-induced forces and their coupling, taking into account the effect of the top tension and internal flow in the riser. The VIV model is solved by combining the Newmark- $\beta$ and Runge-Kutta methods and verified with experimental data from the literature. Combining Option 1 and Option 2 failure assessment diagrams (FADs) in the BS7910 standard, a fracture failure assessment model for a marine riser with circumferential semielliptical outside surface cracks is established. Using the VIV model and FAD failure assessment chart, the effects of riser length, inside/outside flows, and top tension on the VIV response and safety assessment of marine risers with outside surface cracks are investigated. It is shown that increasing the top tension can inhibit the lateral displacement amplitude and bending stress in a riser, but excessive top tension can increase the axial stress in the riser, which counteracts the decrease in the bending stress, so that the effect of top tension on crack safety is not significant. The increasing outside flow velocity significantly increases the lateral vibration amplitude and bending stress in the riser and reduces the crack safety. When other parameters remain unchanged, increasing riser length has no significant effect on the vibration amplitude of the lower part of the riser.
\end{abstract}

\section{Introduction}

The marine riser (Figure 1) is an important pipe connecting the seabed wellhead and the drilling platform, in which a surface crack subjected to alternating stress due to vortex-induced vibration (VIV) will propagate gradually and penetrate the wall, causing fracture and leakage, as well as bringing serious economic loss and safety hazard to offshore oil and gas production. Therefore, evaluating the crack safety in risers is an important aspect of riser design, operation, and maintenance.

Many scholars have studied this problem. Hartlen and Currie [1] established the transverse vibration model of elastically supported cylinders and the corresponding empirical model of fluid force, based on which the VIV analysis is carried out. The fluid force model showed that fluid force satisfies the nonlinear van der Pol equation. Sarpkaya [2] presented a comprehensive discussion of the induced mechanism of VIV, the response mechanism of the fluid model, and the vortex-induced shedding mechanism of fluid flowing through a fixed vertical cylinder. Guo et al. [3] established the VIV differential equation for marine risers and solved it discretely using the Hermit interpolation function. Combining this with Miner's rule, the fatigue life of the riser was analyzed, and it showed that the internal flow of the riser had some influence on the VIV response as well as fatigue life. Gu et al. [4] pointed out that, compared with the traditional finite element method, the generalized integral transformation technique (GITT) for solving the wake oscillator model had a shorter calculation time and smaller error. Measured motions from flexible pipe VIV tests 


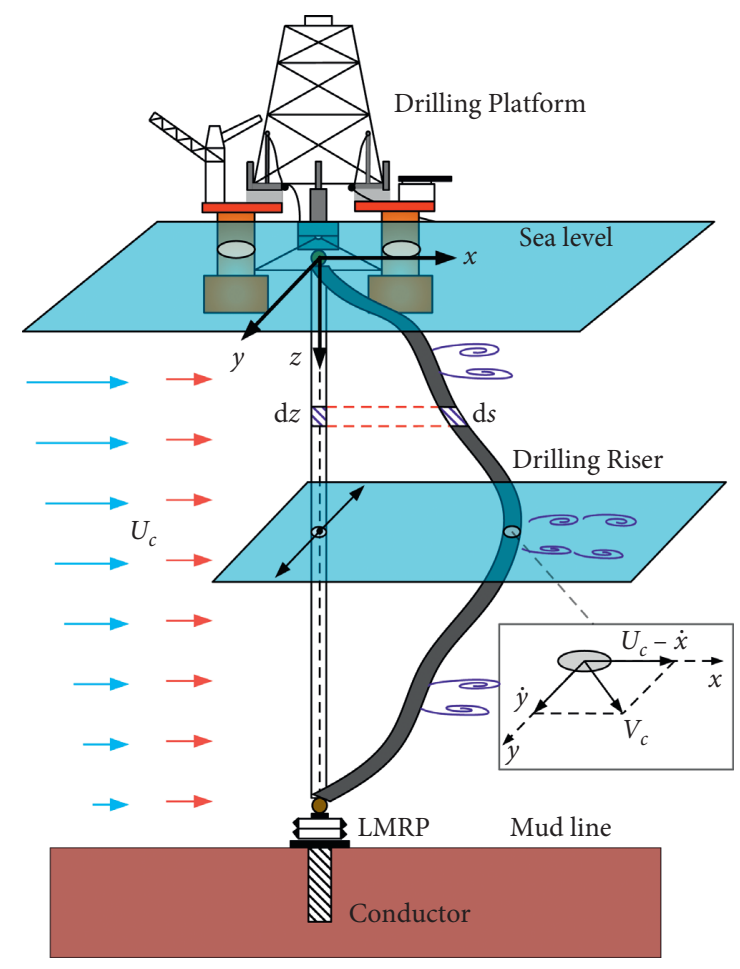

FIgURE 1: Schematic of a marine riser.

contain higher-order harmonic components, which have not yet been extensively studied. Yin et al. [5] and Song et al. [6] presented results from conventional forced-motion VIV experiments but used measured motions taken from a flexible pipe undergoing VIV. Moreover, the comparison between their calculation results and experimental data showed that the wake oscillator model combined with the GITT method can effectively analyze the VIV problem in a marine riser. The GITT method was used by Xu et al. [7] to analyze the VIV time-history response of a marine riser, and it showed that the bending moment caused by vortex-induced force had a great influence on riser life. Lately, some scholars $[8,9]$ have studied the vibration characteristics of marine risers on the basis of considering the coupling effects of downstream and transverse directions. However, no further analysis has been made on the crack growth of risers.

The focus of the above studies was on the VIV and fatigue life of flawless risers. However, the service life of a riser in a marine environment was not taken into account. In manufacturing or transportation, small cracks inevitably occur in risers. In the marine environment, these microcracks propagate gradually until they penetrate the pipe wall under the action of alternating load caused by internal/ external flow. If not managed in a timely manner, they will cause irreversible destructive damage. In view of this, some scholars have established finite element models of marine risers with external surface cracks, considering the effects of wave loads [10], internal pressure [11], ship drift [12], and pure moment loads [13] on the risers. On this basis, the fatigue life of risers under internal and external loads was analyzed. These works show that defects in risers can significantly reduce their fracture strength and fatigue life, and do great harm to their safety and reliability, which requires attention.

In recent years, codes and standards for the safety assessment of offshore pipeline defects have gradually been developed, such as BS7910 [14], R6 [15], DNV-OS-F101 [16], and DNV-RP-F108 [17], of which BS7910 [14] is a mainstream safety assessment standard for pipelines in which the fracture mechanics, the plastic limit load method, and a failure assessment diagram (FAD) are used to evaluate the safety of defective pipelines. Based on the engineering FAD in BS7910 [14], the finite element method was used by El-Sayed et al. [18] to analyze the geometric parameters of a critical crack defect outside the pipe wall under internal pressure. The safety assessment of X70 steel with circumferential buried crack defects was carried out by Li et al. [19] using the 2B assessment method of BS7910 standard, and the applicability of the standard to the assessment of marine riser defects was verified. Tjhen et al. [20] presented a threedimensional finite element model with an external surface crack and studied the evolution of crack opening displacement under pure bending and biaxial loading. Subsequently, based on the BS7910 standard, the FAD for this type of defect was established. Zhang et al. [21] proposed a fracture assessment method for composite pipes under large bending moments and determined the different parameters that affected fracture behavior. Aiming at the crack propagation failure of 3D pipe, the evolution of crack tip opening displacement (CTOD) of the pipeline with semielliptical surface crack in weld girth is investigated, and a way to assess fracture capability of a practical pipeline subject to large plastic deformation was proposed by Yi et al. [22]. Moreover, the influence of corrosion defects parameters on remaining strengths was investigated for girth welded pipelines, and an equation was proposed to evaluate the corrosion progress across girth welded pipelines by Zhang et al. [23].

The above studies discussed the fracture assessment of marine risers with defects under pure bending stress or axial stress. However, in a marine environment, risers bear not only bending stress but also axial stress. Therefore, the safety assessment of risers with defects under a single load may be quite different from the actual situation. In this study, the wake oscillator model is used to simulate the interaction between riser and ocean fluid, based on which the VIV model of a riser is established considering the combined action of top tension, ocean current, and fluid inside the riser. The model is transformed by the GITT method and solved by Gear's BDF method to determine the maximum dynamic bending moment and axial force response of the riser. Then, the FAD of a riser with a circumferential outside surface crack is established according to Option 1 and Option 2 assessments in BS7910 [14], based on which the effects of different pipe lengths, inside flow, outside flow, and top tension on the crack safety of a riser with a circumferential outside surface crack are investigated.

\section{VIV Prediction Model of a Marine Riser}

It is very difficult or even impossible to accurately describe the VIV phenomenon, which is an interaction mechanism between fluid and structure. Therefore, 
empirical formulas have been sought by many researchers to describe this problem, among which the wake oscillator model is the most commonly used. In this model, the wake is viewed as a nonlinear oscillator, and the fluid/oscillating structure is regarded as a whole system. In this section, a VIV model of the marine riser is established by introducing the wake oscillator model into the lateral vibration model of the riser, which takes into account the combined action of top tension, inside flow, and outside flow.

2.1. Establishment and Solution. As shown in Figure 1, the top of the riser is connected to the platform through a spherical joint and an extension component, providing top tension to prevent buckling and excessive axial force. The bottom end is connected to the lower riser lifting kit through a ball socket joint. Therefore, before establishing the VIV model, the following basic assumptions are put forward:

(1) The upper end of the riser is simplified as a simply supported constraint
(2) The lower end of riser is simplified as a hinge constraint

(3) The direction of wave movement is the same as that of the ocean current

(4) The fluid in the riser is assumed as Newtonian fluid and flows at a constant upward speed

(5) The riser material is homogeneous and isotropic

Based on the small deformation hypothesis and the Kirchhoff hypothesis, the three displacement field components $u_{1}, u_{2}$, and $u_{3}$ along the coordinates $x, y$, and $z$, respectively, can be written as

$$
\left\{\begin{array}{l}
u_{1}(z, t)=v_{x}(z, t), u_{2}(z, t)=v_{y}(z, t) \\
u_{3}(z, x, t)=u(z, t)-X \frac{\partial v_{x}}{\partial z}(z, t)-y \frac{\partial v_{y}}{\partial z}(z, t) .
\end{array}\right.
$$

Substitute equation (1) into the following Green strain expression:

$$
\begin{aligned}
& \varepsilon_{x x}=\frac{\partial u_{1}}{\partial x}, \varepsilon_{y y}=\frac{\partial u_{2}}{\partial y}, \varepsilon_{z z}=\frac{\partial u_{3}}{\partial z} \\
& \varepsilon_{x z}=\frac{1}{2}\left(\frac{\partial u_{1}}{\partial z}+\frac{\partial u_{3}}{\partial x}\right), \varepsilon_{y z}=\frac{1}{2}\left(\frac{\partial u_{2}}{\partial z}+\frac{\partial u_{3}}{\partial y}\right), \varepsilon_{x y}=\frac{1}{2}\left(\frac{\partial u_{1}}{\partial y}+\frac{\partial u_{2}}{\partial x}\right) .
\end{aligned}
$$

The riser strain can be written as

$$
\begin{aligned}
& \varepsilon_{z z}=\frac{\partial u}{\partial z}-x \frac{\partial^{2} v_{x}}{\partial z^{2}}-y \frac{\partial^{2} v_{y}}{\partial z^{2}}, \\
& \varepsilon_{x x}=\varepsilon_{y y}=\varepsilon_{x z}=\varepsilon_{y z}=\varepsilon_{x y}=0 .
\end{aligned}
$$

According to equation (3) and the definition of strain energy, the riser strain energy can be expressed as follows:

$$
U_{P}=\frac{1}{2} \int_{0}^{L} E A u^{\prime 2}+E I\left(v_{x}^{\prime \prime 2}+v_{y}^{\prime \prime 2}\right) \mathrm{d} z .
$$

The total kinetic energy of the riser can be expressed as

$$
W_{K}=\frac{1}{2} \int_{0}^{L}\left(m_{v}+m_{a}\right)\left(\dot{v}_{x}^{2}+\dot{v}_{y}^{2}\right)+m_{i}\left(v_{x}^{2}+v_{y}^{2}+v_{z}^{2}\right) \mathrm{d} z
$$

where $v_{x}, v_{y}$, and $v_{z}$ represent the components of the absolute velocity of fluid in the riser along the three coordinate directions $x, y$, and $z$, respectively. The above parameters can be written as follows:

$$
\left\{\begin{array}{l}
m_{v}=\rho_{v} A, m_{a}=C_{m} \rho_{w} \pi \frac{D_{o}^{2}}{4}, m_{i}=\rho_{i} \frac{\pi D_{i}^{2}}{4}, \\
v_{x}=\dot{v}_{x}+V v_{x}^{\prime}, v_{y}=\dot{v}_{y}+V v_{y}^{\prime}, v_{z}=V .
\end{array}\right.
$$

For a riser section with length $\mathrm{dz}$, its length after deformation ds (Figure 1) can be written as follows:

$$
\mathrm{d} s=\sqrt{1+v_{x}^{\prime 2}+v_{y}^{\prime 2}} \mathrm{~d} z .
$$

Therefore, the longitudinal strain and its variation in the riser can be written, respectively, as

$$
\left\{\begin{array}{l}
u^{\prime}=\varepsilon_{0}+\frac{\mathrm{d} s-\mathrm{d} z}{\mathrm{~d} z} \cong \varepsilon_{0}+\frac{1}{2}\left(v_{x}^{\prime 2}+v_{y}^{\prime 2}\right), \\
\delta u^{\prime}=v_{x}^{\prime} \delta v_{x}^{\prime}+v_{y}^{\prime} \delta v_{y}^{\prime} .
\end{array}\right.
$$

The integration forms of $U_{P}$ and $W_{K}$ over time can be expressed as

$$
\begin{aligned}
\int_{t_{i}}^{t_{f}} \wp \mathrm{d} t= & \int_{t_{i}}^{t_{f}} W_{K}-U_{P} \mathrm{~d} t \\
= & \frac{1}{2} \int_{t_{i}}^{t_{f}} \int_{0}^{L}\left(m_{v}+m_{a}\right)\left(\dot{v}_{x}^{2}+\dot{v}_{y}^{2}\right)+m_{i}\left(v_{x}^{2}+v_{y}^{2}+v_{z}^{2}\right) \\
& -E A u^{\prime 2}-E I\left(v_{x}^{\prime \prime 2}+v_{y}^{\prime \prime 2}\right) \mathrm{d} z \mathrm{~d} t .
\end{aligned}
$$

A variation of equation (9) can be written as 


$$
\begin{aligned}
& \delta \int_{t_{i}}^{t_{f}} \wp \mathrm{d} t \\
& \quad=\int_{t_{i}}^{t_{f}} \int_{0}^{L}\left(m_{v}+m_{a}\right)\left(\dot{v}_{x} \delta \dot{v}_{x}+\dot{v}_{y} \delta \dot{v}_{y}\right)+m_{i}\left(\dot{v}_{x}+V v_{x}^{\prime}\right) \delta\left(\dot{v}_{x}+V v_{x}^{\prime}\right) \\
& \quad+m_{i}\left(\dot{v}_{y}+V v_{y}^{\prime}\right) \delta\left(\dot{v}_{y}+V v_{y}^{\prime}\right)-E A u^{\prime} \delta u^{\prime}-E I\left(v_{x}^{\prime \prime} \delta v_{x}^{\prime \prime}+v_{y}^{\prime \prime} \delta v_{y}^{\prime \prime}\right) \mathrm{d} z \mathrm{~d} t,
\end{aligned}
$$

where $T_{e}\left(=E A u^{\prime}\right)$ denotes the effective axial force of the riser unit.

Using equations (8) and (10), it can be further written as

$$
\begin{aligned}
\delta \int_{t_{i}}^{t_{f}} \wp \mathrm{d} t & \\
= & \int_{t_{i}}^{t_{f}} \int_{0}^{L}\left[\begin{array}{c}
-\left(m_{v}+m_{a}\right) \ddot{v}_{x}-m_{i}\left(\ddot{v}_{x}+2 V \dot{v}_{x}^{\prime}+V^{2} v_{x}^{\prime \prime}\right) \\
+T_{e} v_{x}^{\prime \prime}-E I v_{x}^{\prime \prime \prime}
\end{array}\right] \delta v_{x} \\
& +\left[\begin{array}{c}
-\left(m_{v}+m_{a}\right) \ddot{v}_{y}-m_{i}\left(\ddot{v}_{y}+2 V \dot{v}_{y}^{\prime}+V^{2} v_{y}^{\prime \prime}\right) \\
+T_{e} v_{y}^{\prime \prime}-E I v_{y}^{\prime \prime \prime}
\end{array}\right] \delta v_{y} \mathrm{~d} z \mathrm{~d} t .
\end{aligned}
$$

The virtual work done by structural damping and external hydrodynamic forces is given as

$$
\delta W=\int_{0}^{L}\left(F_{D}-F_{c x}\right) \delta v_{x}+\left(F_{L}-F_{c y}\right) \delta v_{y} \mathrm{~d} z,
$$

where $F_{c x}$ and $F_{c y}$ are structural damping forces and $F_{D}$ and $F_{L}$ are the drag force in the inline flow (IL) direction and the lateral lift in the transverse flow direction, respectively. The four forces are further written as follows:

$$
\left\{\begin{array}{l}
F_{c x}=c \dot{v}_{x}, F_{c y}=c \dot{v}_{y}, c=2 m \omega \xi, \omega=\pi^{2} \sqrt{\frac{E I}{m L^{4}}} \\
F_{D}=\frac{1}{2} \rho_{w} D_{o} C_{D} V_{r}\left(U_{c}-\dot{v}_{x}\right) \\
F_{L}=\frac{1}{2} \rho_{w} D_{o} C_{L}\left(U_{c}-\dot{v}_{x}\right)^{2}-\frac{1}{2} \rho_{w} D_{o} C_{D} V_{r} \dot{v}_{y}
\end{array},\right.
$$

where $m=m_{v}+m_{a}+m_{i}$.

According to Hamilton's principle $\delta \int_{t_{i}}^{t_{f}} \wp+W \mathrm{dt}=0$ and equations (11)-(13), the vibration equations of the riser in the $x$ and $y$ directions can be given as follows:

$$
\left\{\begin{array}{l}
\left(m_{v}+m_{a}+m_{i}\right) \ddot{v}_{x}+c \dot{v}_{x}+2 m_{i} V \dot{v}_{x}^{\prime}+m_{i} V^{2} v_{x}^{\prime \prime}-T_{e} v_{x}^{\prime \prime}+E I\left(v_{x}^{\prime \prime}\right)^{\prime \prime}=F_{D} \\
\left(m_{v}+m_{a}+m_{i}\right) \ddot{v}_{y}+c \dot{v}_{y}+2 m_{i} V \dot{v}_{y}^{\prime}+m_{i} V^{2} v_{y}^{\prime \prime}-T_{e} v_{y}^{\prime \prime}+E I\left(v_{y}^{\prime \prime}\right)^{\prime \prime}=F_{L}
\end{array} .\right.
$$

The boundary conditions of the upper and lower ends of the riser are considered to be simply supported:

$$
\left\{\begin{array}{l}
v_{x}(0, t)=0, \frac{\partial^{2} v_{x}(0, t)}{\partial^{2} z}=0, v_{y}(0, t)=0, \frac{\partial^{2} v_{y}(0, t)}{\partial^{2} z}=0 \\
v_{x}(L, t)=0, \frac{\partial^{2} v_{x}(L, t)}{\partial^{2} z}=0, v_{y}(L, t)=0, \frac{\partial^{2} v_{y}(L, t)}{\partial^{2} z}=0
\end{array} .\right.
$$

The van der Pol nonlinear vibration equation is used to describe the shedding characteristics of a vortex in the crossflow (CF) direction. According to a discussion about the wake vibrator coupling term in Facchinetti and Langer [24], as the acceleration coupling term is selected, the VIV calculated results are in good agreement with the experimental results. Based on this idea, the governing equations of wake oscillators can be written as

$$
\left\{\ddot{q}_{x}+\varepsilon_{x} \omega_{s}\left(q_{x}^{2}-1\right) \dot{q}_{x}+\left(2 \omega_{s}\right)^{2} q_{x}=\frac{A_{x}}{D_{i}} \ddot{v}_{x} \ddot{q}_{y}+\varepsilon_{y} \omega_{s}\left(q_{y}^{2}-1\right) \dot{q}_{y}+\left(\omega_{s}\right)^{2} q_{y}=\frac{A_{y}}{D_{i}} \ddot{v}_{y},\right.
$$

where $q_{x}$ and $q_{y}$ are the dimensionless wake oscillator variables in the inline and transverse flow directions. $\omega_{s}=2 \pi S_{t}\left|U_{c}-\dot{v}_{x}\right| / D_{o}$, and $\varepsilon_{x}, \varepsilon_{y}, A_{x}$, and $A_{y}$ are dimensionless parameters determined by the experiment.

Drag coefficient $C_{D}$ and lift coefficient $C_{L}$ can be expressed by the wake oscillator variables $q_{x}$ in the IL direction and $q_{y}$ in the $\mathrm{CF}$ direction as follows:

$$
\left\{\begin{array}{l}
C_{D}=C_{D 0} A_{a}+C_{i 0} \frac{q_{x}}{2} \\
C_{L}=C_{L 0} \frac{q_{y}}{2}
\end{array}\right.
$$

where $A_{a}$ is the drag force amplification factor, for which the calculation formula was presented by Vandiver [25]: 


$$
A_{a}=1+1.043\left(\frac{2 y_{\mathrm{rms}}}{D_{o}}\right)^{0.65}
$$

The VIV control equations for the marine riser are obtained by integrating equations (14)-(18) and making $c^{\prime}=1 / 2 \rho_{w} D_{o} C_{D} V_{r}$.

$$
\left\{\begin{array}{l}
m \ddot{v}_{x}+\left(c+c^{\prime}\right) \dot{v}_{x}+2 m_{i} V \dot{v}_{x}^{\prime}+E I\left(v_{x}^{\prime \prime}\right)^{\prime \prime}-T_{e} v_{x}^{\prime \prime}+m_{i} V^{2} v_{x}^{\prime \prime}=\frac{1}{2} \rho_{w} D_{o} C_{D} V_{r} U_{c} \\
\ddot{q}_{x}+\varepsilon_{x} \omega_{s}\left(q_{x}^{2}-1\right) \dot{q}_{x}+\left(2 \omega_{s}\right)^{2} q_{x}=\frac{A_{x}}{D_{O}} \ddot{v}_{x} \\
m \ddot{v}_{y}+\left(c+c^{\prime}\right) \dot{v}_{y}+2 m_{i} V \dot{v}_{y}^{\prime}+E I\left(v_{y}^{\prime \prime}\right)^{\prime \prime}-T_{e} v_{y}^{\prime \prime}+m_{i} V^{2} v_{y}^{\prime \prime}=\frac{1}{2} \rho_{w} D_{o} C_{L}\left(U_{c}-\dot{v}_{x}\right)^{2} \\
\ddot{q}_{y}+\varepsilon_{y} \omega_{s}\left(q_{y}^{2}-1\right) \dot{q}_{y}+\left(\omega_{s}\right)^{2} q_{y}=\frac{A_{y}}{D_{O}} \ddot{v}_{y}
\end{array} .\right.
$$

The system discretized equations are numerically solved using the Newmark- $\beta$ method. The detailed derivation can be seen in our previous research work (Liu et al. [26]). The wake vibrator is solved by a fourth-order Runge-Kutta technique (Liu et al. [27]). The coupling iteration between them is calculated to determine the dynamic response.

\subsection{Safety Evaluation Method of Surface Crack. Option 1 and} Option 2 assessments in BS7910 [14] are used to evaluate the crack safety of a riser with a circumferential outside surface crack. In BS7910, which is based on the fracture mechanics and the plastic limit load method, the FAD is used to evaluate cracked components, where the brittle fracture ratio $K_{r}$ and plastic collapse ratio $L_{r, \max }$ are set as ordinate and abscissa, respectively, and the safety and unsafe zones are divided according to the plastic instability boundaries. On this basis, the fracture performance of a component with a crack is investigated by judging whether the value of the assessment point falls into the safety area.

In BS7910 [14], the fracture mechanics assessment of a defect is divided into three grades: Option 1, Option 2, and Option 3. Option Grade 1 assessment is a simplified evaluation curve, which is applicable to a low level of information. Option 2 level assessment requires that the relationship between the true stress-strain curve of the structure and its assessment curve has greater accuracy. Option 3 is suitable for ductile materials with stable tearing. Because it requires the calculation of the J-integral of defective structures, it is not used for common structural steels. Therefore, in this study, the first two grade evaluation curves are used to evaluate a riser with a crack defect.

In Option 1, the assessment does not require the true stress-strain curve of the material but only requires the yield strength, Young's modulus, Poisson's ratio, and the impact energy of the material. The description of the evaluation curve is as follows:

$$
\begin{aligned}
& f\left(L_{r}\right)=\left(1+\frac{1}{2} L_{r}^{2}\right)^{-1 / 2}\left[0.3+0.7 * \exp \left(-\mu L_{r}^{6}\right)\right], \quad L_{r} \leq 1, \\
& f\left(L_{r}\right)=f(1) L_{r}^{(N-1) / 2 N}, \quad 1<L_{r}<L_{r, \max }, \\
& f\left(L_{r}\right)=0, \quad L_{r}>L_{r, \max } .
\end{aligned}
$$

where $\quad \mu=\min \left(0.001 E / \sigma_{b}, 0.6\right), \quad N=0.3\left(1-\sigma_{b} / \sigma_{s}\right)$, $L_{r, \max }=\left(\sigma_{s}+\sigma_{b}\right) / 2 \sigma_{s}$.

In Option 2, the failure assessment curve is used to evaluate the safety of a pressure pipe with welded defects where the strength mismatch is more than $10 \%$. The expression is given as follows:

$$
\begin{aligned}
& f\left(L_{r}\right)=\left(\frac{E \varepsilon_{r e f}}{L_{r} \sigma_{s}}+\frac{L_{r}^{3} \sigma_{s}}{2 E \varepsilon_{r e f}}\right)^{-1 / 2}, \quad L_{r}<L_{r, \max }, \\
& f\left(L_{r}\right)=0, \quad L_{r} \geq L_{r, \text { max }},
\end{aligned}
$$

where $\sigma_{r e f}=L_{r} \sigma_{s}$.

The plastic collapse ratio $L_{r}$ is defined as

$$
L_{r}=\frac{\sigma_{r e f}}{\sigma_{s}} .
$$

The brittle fracture ratio $K_{r}$ is defined as

$$
K_{r}=\frac{K_{1}^{p}+K_{1}^{s}}{K_{\text {mat }}},
$$

where $K_{\text {mat }}=\sqrt{E J_{\mathrm{mat}} / 1-\nu^{2}}$.

The general formula for the stress intensity factor is

$$
K_{I}=(Y \sigma) \sqrt{\pi a},
$$

in which the stress intensity factor is composed of major and minor stresses: 


$$
\begin{aligned}
Y \sigma= & (Y \sigma)_{p}+(Y \sigma)_{s}, \\
(Y \sigma)_{p}= & M f_{w}\left\{k_{t m} M_{k m} M_{m} P_{m}\right. \\
& +k_{t b} M_{k b} M_{b}\left[P_{m}+\left(k_{m}-1\right) P_{b}\right\} \\
(Y \sigma)_{s}= & M_{m} Q_{m}+M_{b} Q_{b} .
\end{aligned}
$$

Their detailed calculation formulas can be found in BS7910.

In this paper, the transverse displacement response of the riser that is induced by VIV is obtained by solving equation (14). Then, using the transverse displacement, equation (19) is used to obtain the bending stress and axial stress, based on which the failure assessment calculation model for a marine riser with a circumferential outside surface crack is established.

2.3. Model Verification. The experimental data presented by Song et al. [6] is used to verify the validity of the VIV model and the calculation code in this paper. The VIV experiment of Song et al. was carried out in the towing pool of Shanghai Institute of Ship Science (Figure 2), in which the effect of uniform flow on the riser string was simulated by dragging the flexible riser model horizontally at a constant velocity. The VIV response characteristics of the riser are analyzed by measuring the motion track and strain. The main experimental parameters are shown in Table 1.

Figures 3 and 4 show the RMS distribution of riser displacement with a reduced velocity of 5.87 and 41.12. Figure 3 shows that when the reduced velocity of the riser is 5.87 , the numerical results of the model and the experiment show first-order vibration modes in the CF vibration and IL vibration. The RMS distribution of the riser displacement obtained by the model in this paper is in good agreement with the RMS distribution of the riser displacement obtained by the experiment for the CF vibration and IL vibration. Figure 4 shows that when the reduced velocity of the riser is 41.12 , the vibration modes obtained by the model in this paper and the experiment are fifth-order modes in the CF vibration, while in the IL vibration, the vibration modes obtained from the model and the experiment are seventhorder modes. As for the RMS amplitude of the riser displacement, it can be seen from Figure 4(a) that the numerical results of the model in this paper are different from the experimental results at both ends of the riser, but the closer they are to the middle, the closer they are to each other. From Figure 4(b), it is apparent that the amplitudes of the two models in the downstream direction of the riser are basically the same.

\section{Results and Discussions}

In this section, the response characteristics and crack safety of a typical riser with a circumferential outside surface crack are analyzed. The riser and marine environmental parameters are shown in Table 2, and they are consistent with the parameters presented [28].

\begin{tabular}{|c|c|c|c|}
\hline Parameter & Value & Parameter & Value \\
\hline $\begin{array}{l}\text { Outside diameter } \\
(\mathrm{m})\end{array}$ & 0.030 & Mass ratio & 2.5 \\
\hline Depth of water (m) & 7.9 & Axial stiffness $(\mathrm{N})$ & $1.45 E 7$ \\
\hline Inside diameter $(\mathrm{m})$ & 0.027 & $\begin{array}{l}\text { Structural damping } \\
\text { ratio }\end{array}$ & 0.3 \\
\hline $\begin{array}{l}\text { Young's modulus } \\
\text { (Pa) }\end{array}$ & $1.08 E 11$ & Top tension $(\mathrm{N})$ & 2943 \\
\hline $\begin{array}{l}\text { Length/diameter } \\
\text { ratio }\end{array}$ & 263 & $\begin{array}{l}\text { Mass per unit length } \\
(\mathrm{kg} / \mathrm{m})\end{array}$ & 1.768 \\
\hline $\begin{array}{l}\text { Bending stiffness } \\
\left(\mathrm{N} / \mathrm{m}^{2}\right)\end{array}$ & 1476.763 & Natural frequency $(\mathrm{Hz})$ & 2.27 \\
\hline
\end{tabular}

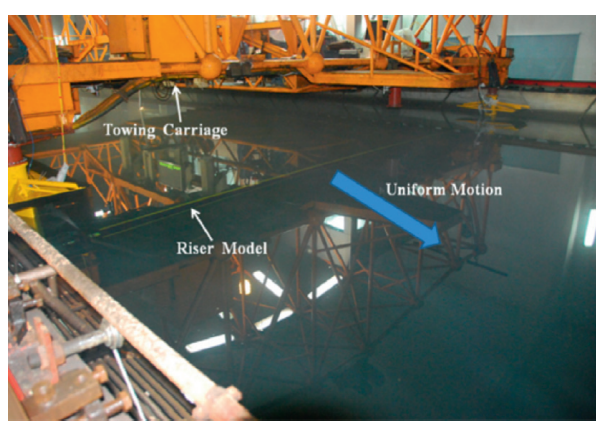

FIgURE 2: Testing setup of a riser in a uniform flow: (a) CF; (b) IL.

TABle 1: Parameters of the riser model in experiment.

3.1. Effect of Top Tension. In order to analyze the influence of different factors on the amplitude of vortex-induced vibration of riser, the response charts for reduced velocity $\left(U_{r}\right)$ clearly reflect the response characteristics of the riser. The reduced velocity $\left(U_{r}\right)$ is defined as

$$
U_{r}=\frac{U}{f_{n} D_{O}},
$$

where $U$ represents the outflow velocity and $f_{n}$ represent the natural frequency of the riser.

The top tension is mainly determined by the structure of the riser and the top tension coefficient $K$ (shown in equation (27)). When investigating the influence of the top tension on the VIV of the riser, this study ensured that the structure of the riser itself remained unchanged and the top tension coefficient changed.

$$
T=A g\left(\rho_{v}-\rho_{w}\right) K L .
$$

Figures 5 and 6 show the maximum amplitude envelope and maximum bending stress envelope of a riser with length $L=300 \mathrm{~m}$ under the conditions of inside flow velocity $V=10 \mathrm{~m} / \mathrm{s}$, different top tensions, and constant outside flow $(U=1.0 \mathrm{~m} / \mathrm{s})$, but the reduced velocity varies with the natural frequency. Figure 5 shows that the maximum vibration amplitude of the riser appears near the upper and lower joints. In the case of small top tension, the order of the vibration mode in the middle of the riser is higher than that in other parts. With the increase in top tension, the order of 


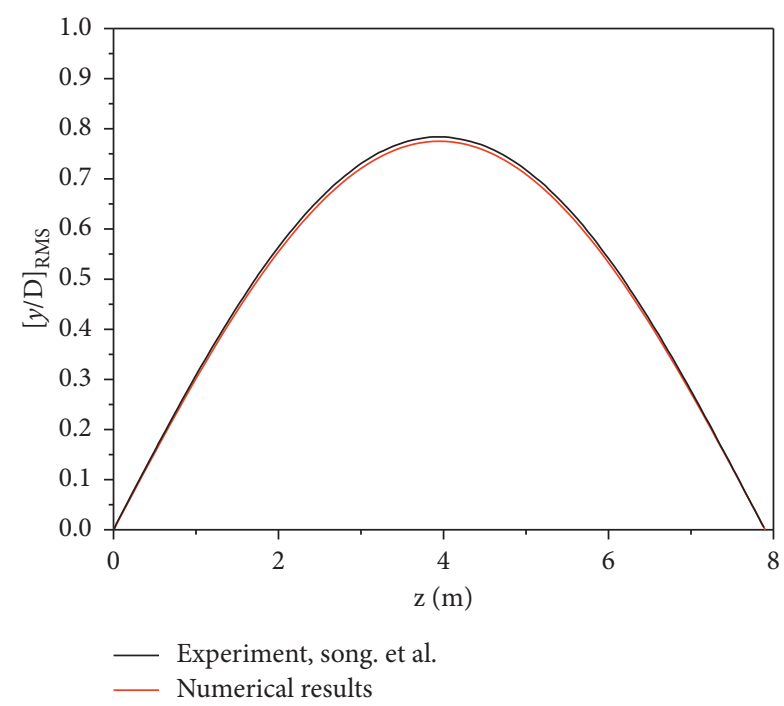

(a)

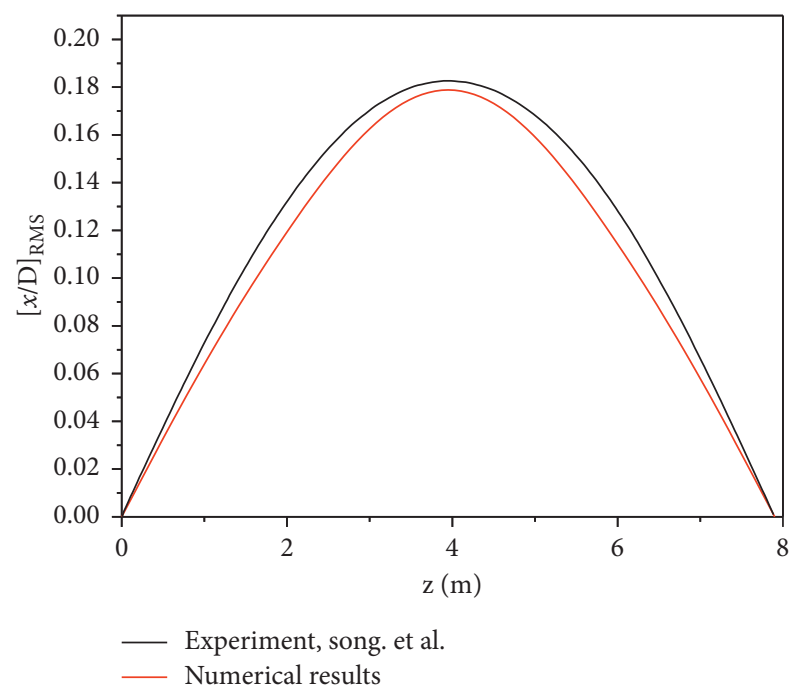

(b)

FIGURE 3: Verification diagram of RMS distribution of riser displacement with reduced velocity of 5.87: (a) CF; (b) IL.

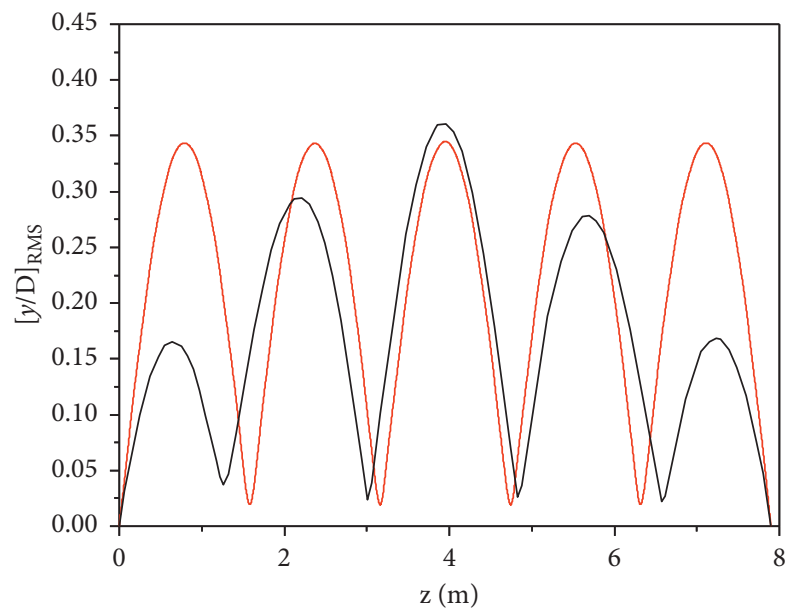

Experiment, song. et al. Numerical results

(a)

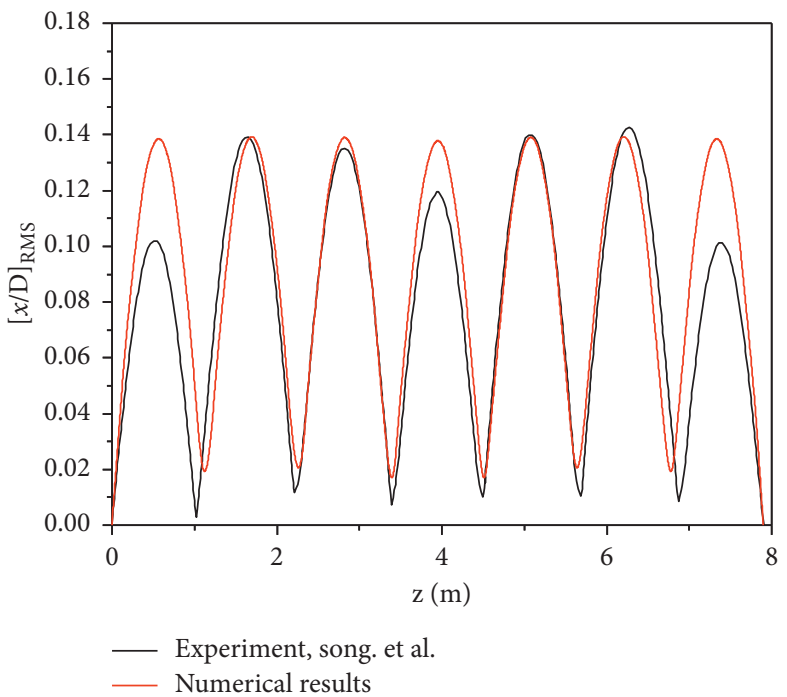

(b)

Figure 4: Verification diagram of RMS distribution of riser displacement with reduced velocity of 41.12: (a) CF; (b) IL.

TABle 2: Properties and size parameters of X65 material for pipeline steel.

\begin{tabular}{|c|c|c|c|}
\hline Parameter & Value & Parameter & Value \\
\hline Outside diameter $(\mathrm{m})$ & 0.5588 & Flow stress $(\mathrm{MPa})$ & 526 \\
\hline Thickness (m) & 0.00127 & Crack semiangle & 0.785 \\
\hline Outside flow velocity $(\mathrm{m} / \mathrm{s})$ & $0.5,1.0,1.5,2.0$ & Inside fluid density $\left(\mathrm{kg} / \mathrm{m}^{3}\right)$ & 1200 \\
\hline Structural damping ratio $(\%)$ & 0.3 & Pipe material density $\left(\mathrm{kg} / \mathrm{m}^{3}\right)$ & 7900 \\
\hline Yield strength of base metal (MPa) & 478 & Yield strength of welding seam (MPa) & 486 \\
\hline Tensile strength of base metal $(\mathrm{MPa})$ & 572 & Tensile strength of welding seam (MPa) & 587 \\
\hline Sea water density $\left(\mathrm{kg} / \mathrm{m}^{3}\right)$ & 1025 & Additional mass coefficient & 1.0 \\
\hline Inside flow velocity $(\mathrm{m} / \mathrm{s})$ & $0,10,20,40$ & Crack depth $(\mathrm{mm})$ & 0.002 \\
\hline Poisson ratio & 0.3 & Crack length (mm) & 0.01 \\
\hline Young's modulus (GPa) & 207 & Strain index & 4.33 \\
\hline Riser length (m) & $300,400,500,600$ & Strain constant & 4.06 \\
\hline Coefficient of top tension & $1.2,1.3,1.4,1.5$ & Internal pressure $(\mathrm{MPa})$ & 10 \\
\hline
\end{tabular}




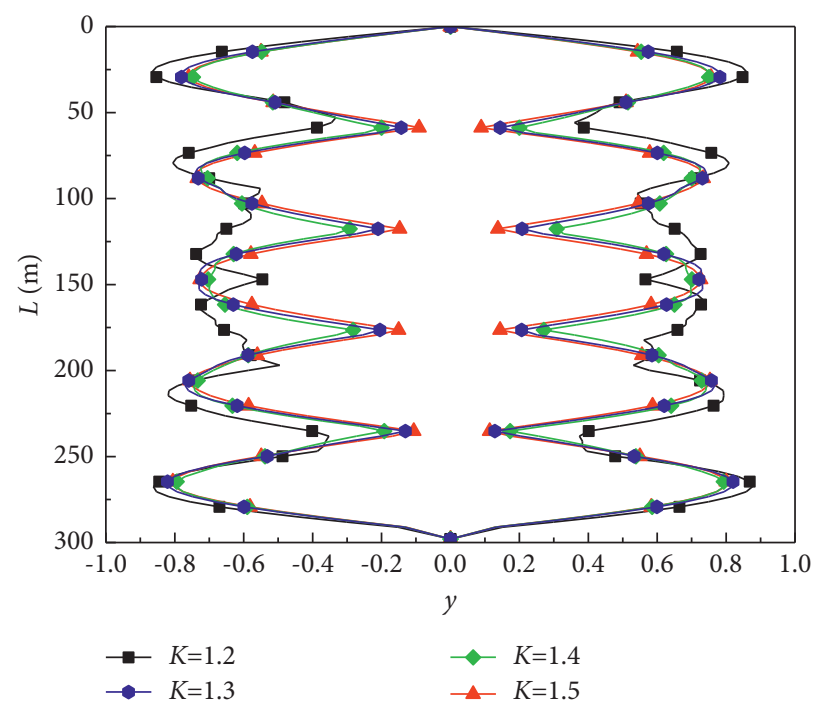

Figure 5: VIV amplitude envelope diagram of the riser under different top tensions.

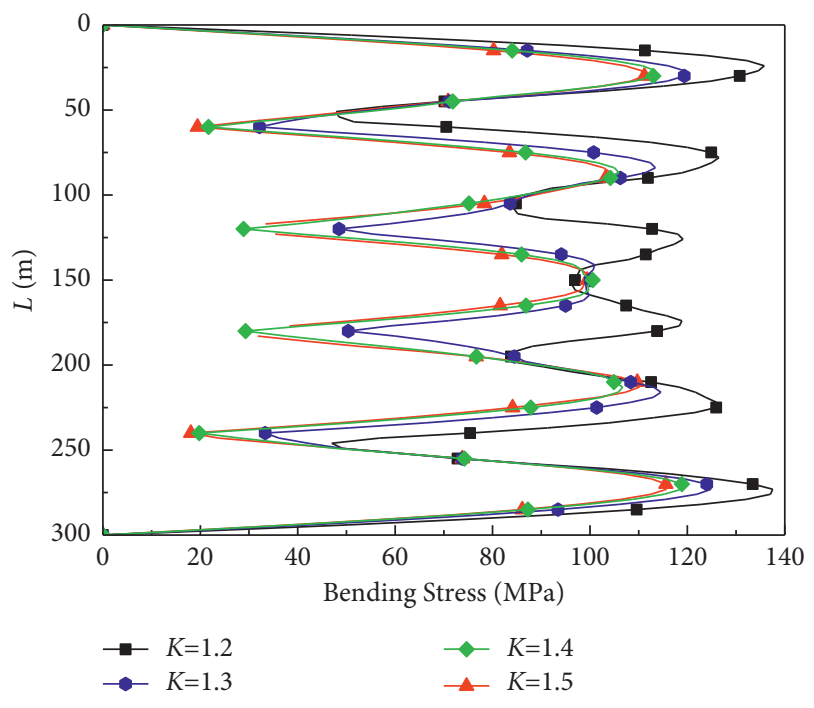

FIGURE 6: VIV bending stress distribution of the riser under different top tensions.

vibration decreases. When the top tension coefficient $K$ increases from 1.3 to 1.5 , there is no obvious change in the vibration form at the upper and lower joints of the riser. However, the vibration order and amplitude in the middle of the riser decrease accordingly. With a further increase in $K$, the vibration order does not change, and its amplitude decreases slightly. Figure 6 shows that the bending stress amplitude is largest at both ends of the riser, and the closer to the middle of the riser, the smaller the bending stress amplitude. With the increase in top tension, the bending stress decreases. However, when the top tension coefficient exceeds 1.3 , the bending stress varies little with the top tension. It is noteworthy that with the increase in top tension, the axial stress in the riser also increases, increasing the load strength of the riser. Therefore, it is more reasonable to set the top tension coefficient to 1.3 .
Figure 7 shows the FAD of the riser under different top tensions, in which the assessment point is located at the position where the bending stress is the largest in Figure 5. As shown in the figure, the fracture assessment points of the cracked riser corresponding to different top tensions are concentrated near a point $(0.412,0.274)$ within the failure assessment curves of Option 1 and Option 2, and there is a large safety margin, which shows that the top tension has little effect on the fracture performance of risers with cracks. The main reason for this is that the increase in top tension increases the axial force and reduces the bending moment in the riser. The two forces cancel each other out, with the result that the top tension has little effect on the fracture performance of cracks in the riser.

3.2. Effect of Inside Flow Velocity. Figures 8 and 9 show the maximum amplitude envelope diagram and the maximum bending stress envelope diagram of a riser with length $L=$ $300 m$ under top tension coefficient $K=1.3$ and different inside flow velocities, and the reduced velocity varies with the natural frequency. Figure 8 shows that the vibration order of the riser increases with the increasing inside flow velocity. When the internal flow velocity increases, the amplitude in the middle of the riser changes slightly, the amplitude in the upper riser decreases dramatically, and the amplitude in the lower riser increases slightly. Therefore, under the condition of high internal velocity, the amplitude of the riser appears trapezoidal in shape with a narrow top and wide bottom.

Figure 9 shows that the distribution of the bending stress amplitude in the riser is more uniform at low internal flow velocity. With the increase in inside flow velocity, the bending stress in the riser increases more obviously, and the farther away from the water surface, the more significant the increase in bending stress.

Figure 10 shows the FAD of risers at different internal flow velocities, in which the assessment point is located at 


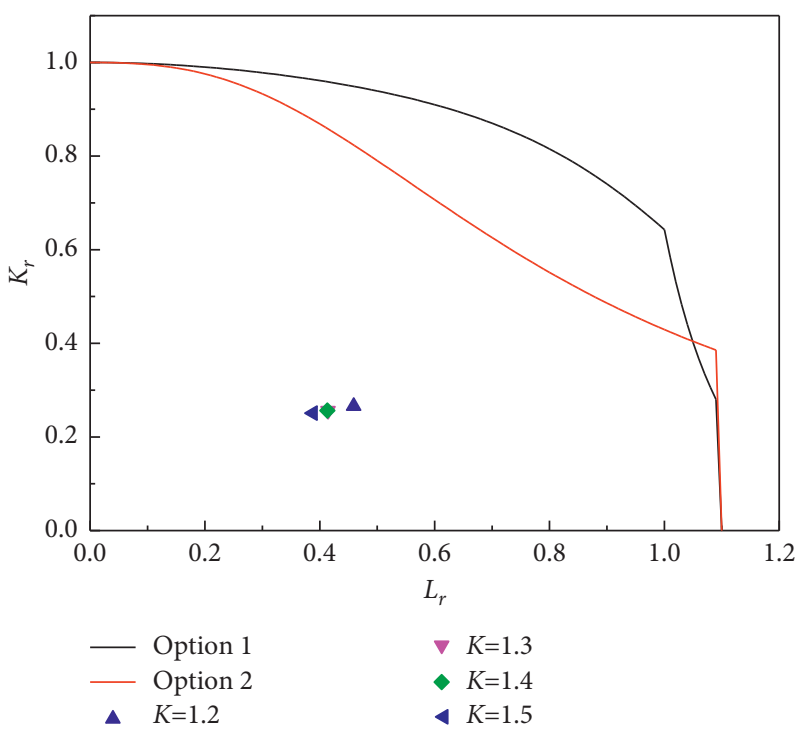

FIGURE 7: Effect of the top tension on the fracture performance of the riser.

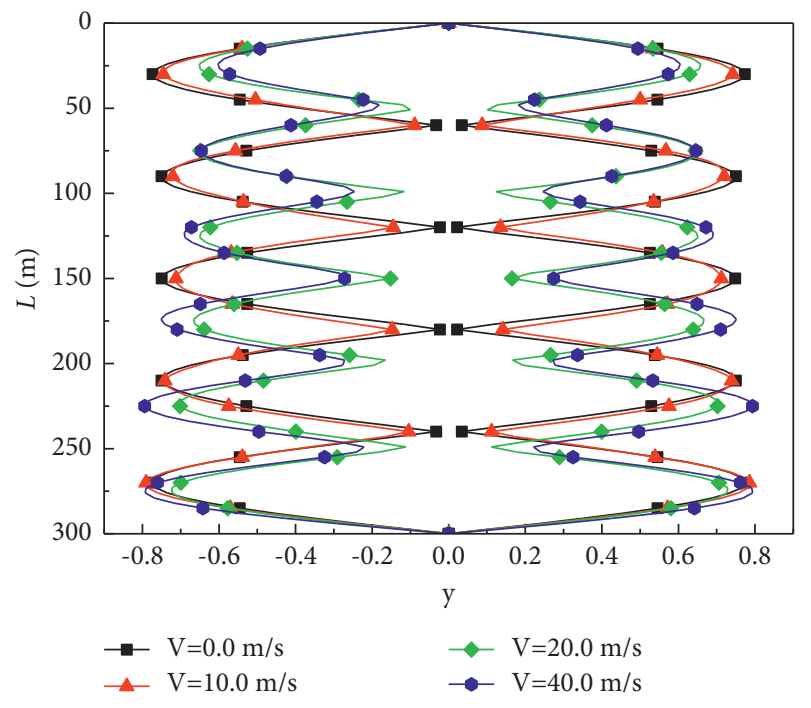

FIGURE 8: VIV amplitude envelope of risers under different internal flow velocities.

the position where the bending stress is the largest in Figure 9. With the increase in internal flow velocity, the safety of cracks at the assessment points gradually decreases.

3.3. Effect of the Reduced Velocity. Figures 11 and 12 show the maximum amplitude envelope diagram and the maximum bending stress envelope diagram of the riser with length $L=300 \mathrm{~m}$ under top tension coefficient $K=1.3$, inside flow velocity $V=10.0 \mathrm{~m} / \mathrm{s}$, and different reduced velocities.

Figure 11 shows that, with the increasing outside flow velocity, the order of the riser vibration decreases gradually, the amplitude of the upper riser increases, and the amplitude of the middle and lower riser changes slightly. Figure 12 shows that the bending stress in the riser decreases

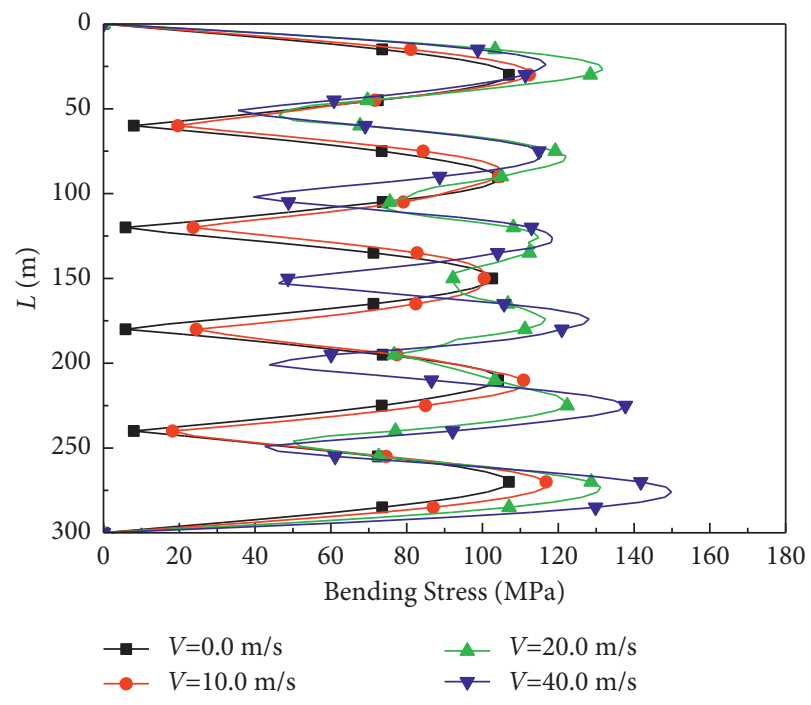

Figure 9: VIV bending stress distribution of riser under different internal flow velocities.

significantly with the increase in reduced velocity. At high reduced velocity, the bending stress amplitude in the riser is distributed uniformly along the length of the riser. At low reduced velocity, the bending stress near the upper and lower ends of the riser is large, while the bending stress in the middle is small. Figure 13 shows the FAD of risers at different reduced velocities, in which the assessment point is located at the position where the bending stress is the largest in Figure 12. It can be seen from the figure that the safety of cracks decreases significantly with the decrease in reduced velocity.

3.4. Effect of Pipe Length. Figures 14 and 15 show the maximum amplitude envelope diagram and the maximum bending stress envelope diagram of the riser under top tension coefficient $K=1.3$, external flow velocity $U=1.0 \mathrm{~m} / \mathrm{s}$, 


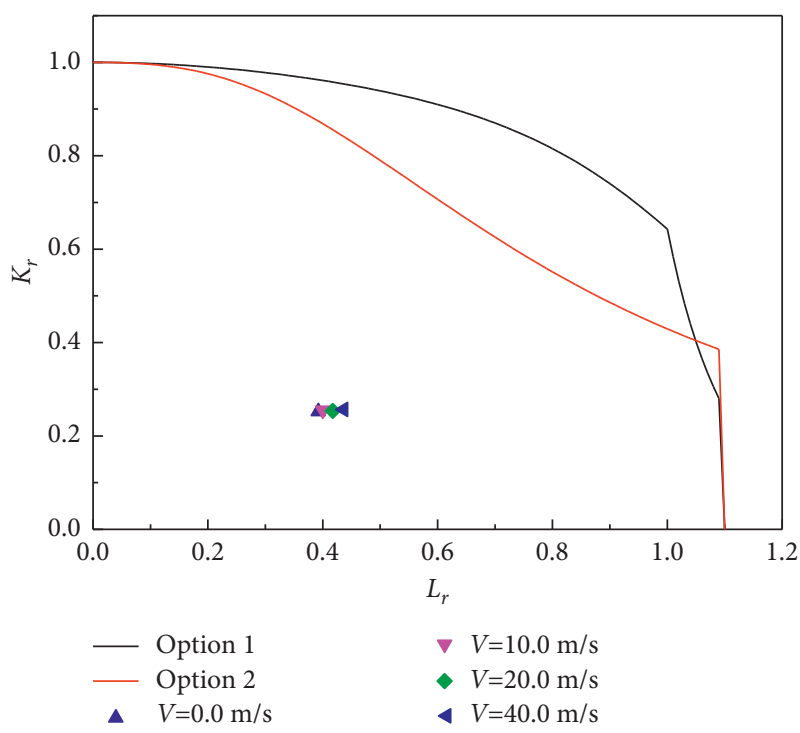

Figure 10: Effect of the top tension on the fracture performance of the riser.

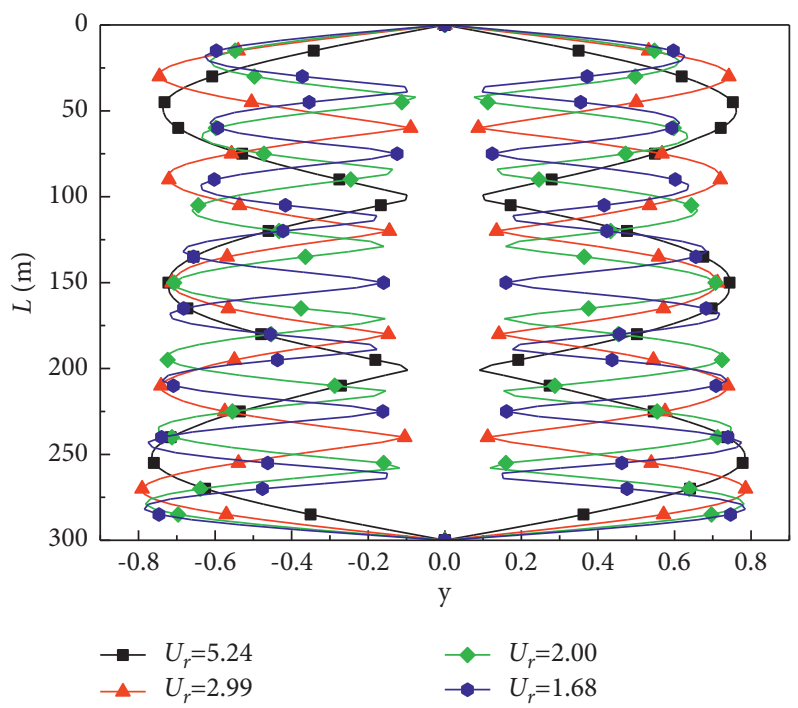

Figure 11: The VIV amplitude envelope of risers under different reduced velocities.

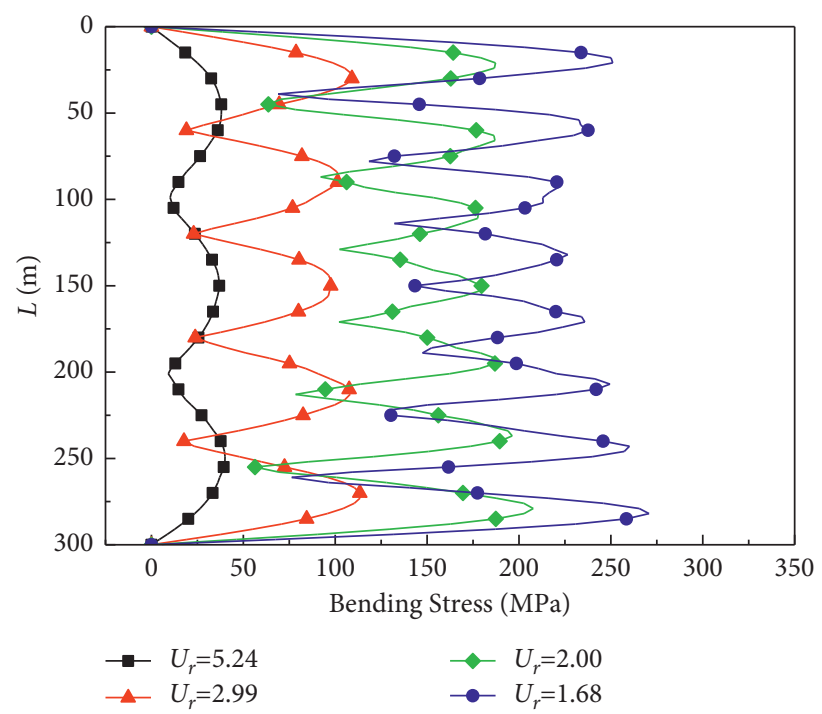

FIGURE 12: VIV bending stress distribution of riser under different reduced velocities. 


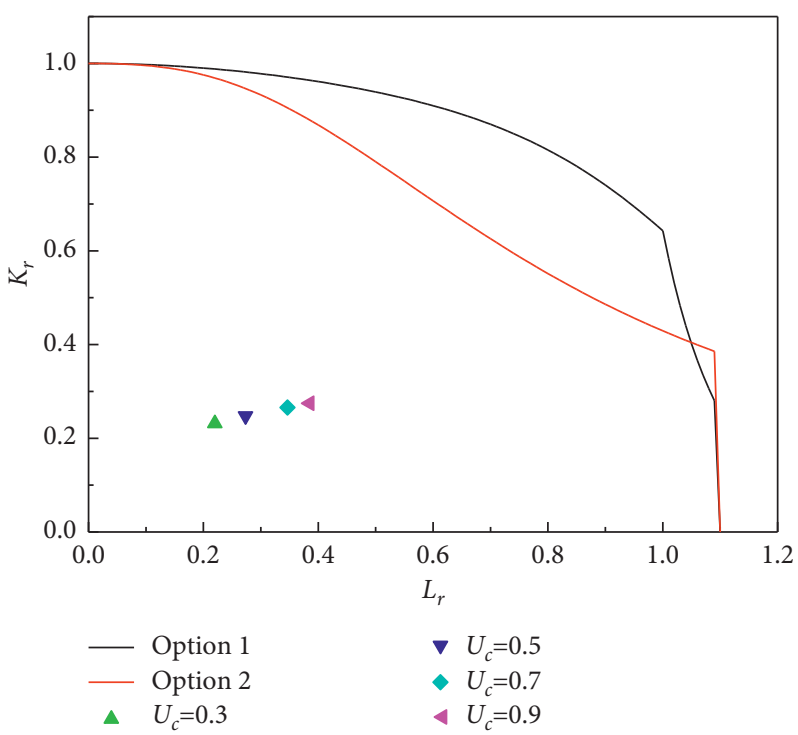

FIGURE 13: Effect of the reduced velocity on the fracture performance of the riser.

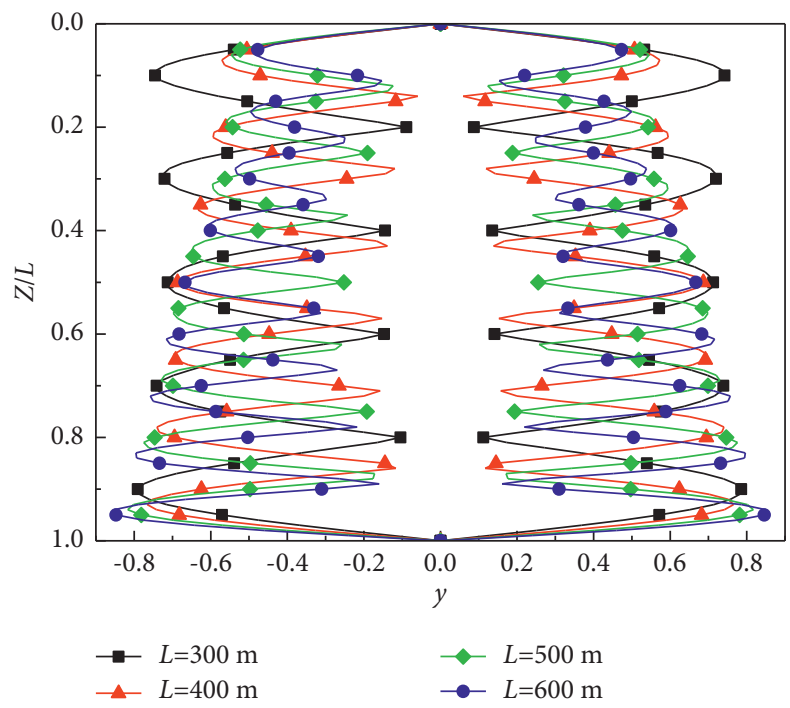

FIgURE 14: The VIV amplitude envelope of riser under different riser lengths.

internal flow velocity $V=10.0 \mathrm{~m} / \mathrm{s}$, different riser lengths, and different reduced velocities by pipe length.

Figure 14 shows that, with the increase in pipe length, the vibration order of the riser increases, and the amplitude of the upper riser decreases significantly, while the amplitude of the middle and lower riser changes slightly. Figure 15 shows that the bending stress in the riser decreases significantly with the increase in riser length. Figure 16 shows the FAD of risers at different internal flow velocities, in which the assessment point is located at the position where the bending stress is the largest in Figure 15.

It can be seen from the diagram that the longer the riser is, the more the crack is inclined to be unsafe, but this effect is not significant. 


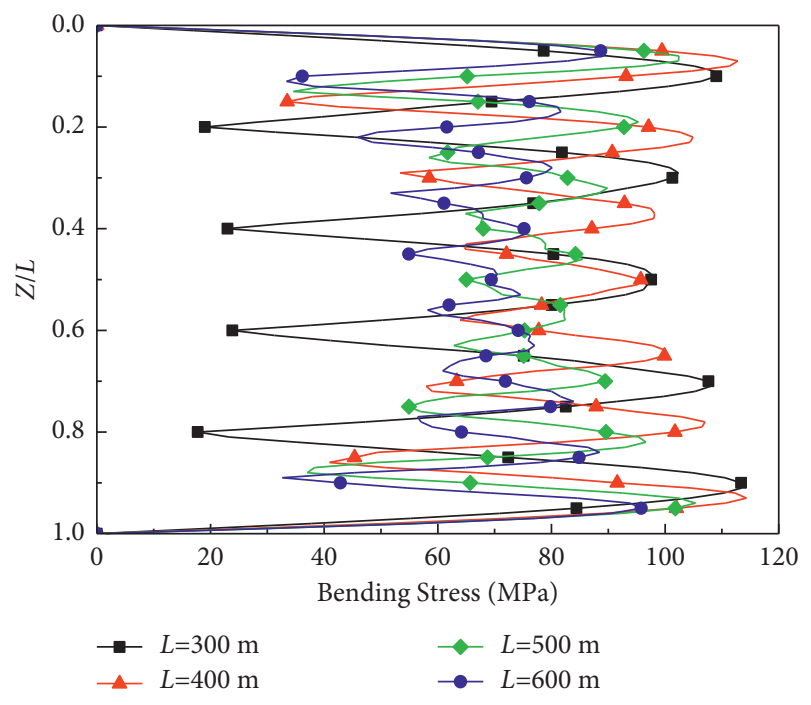

FIGURE 15: VIV bending stress distribution of riser under different riser lengths.

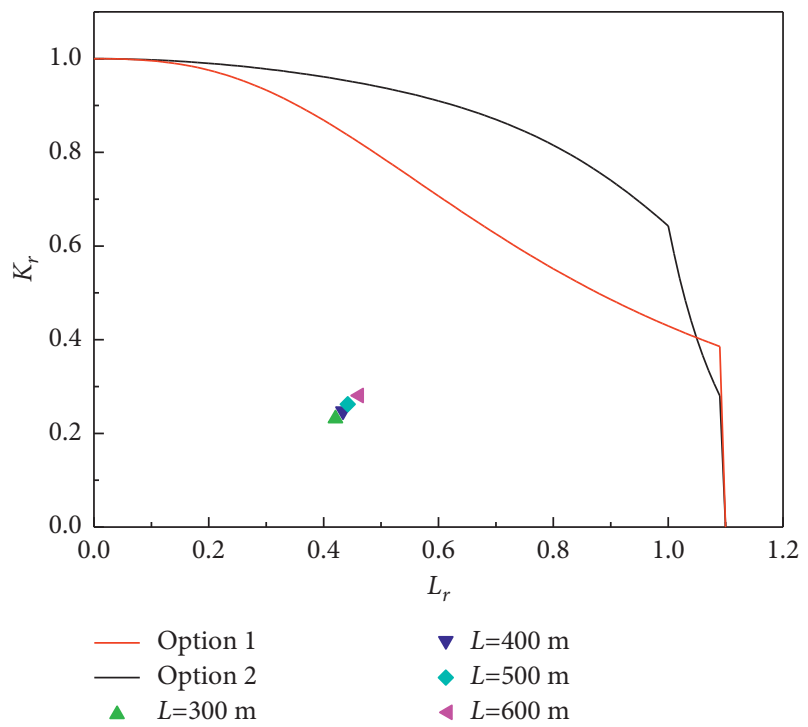

FIGURE 16: Effect of the riser length on the fracture performance of the riser.

\section{Conclusion}

A nonlinear VIV model combined with a crack assessment technique for a marine riser was established to investigate the effects of riser length, inside/outside flows, and top tension on the VIV response and crack safety of marine risers. The following conclusions can be drawn:

(1) Increasing the top tension properly can effectively reduce the amplitude and bending stress of a riser. However, as the top tension coefficient exceeds 1.3, the effect of top tension becomes limited. At the same time, excessive top tension will increase the axial stress in the riser and reduce crack safety. Crack evaluation analysis shows that the top tension has no significant effect on crack safety. The main reason for this is that when the top tension changes, the decrease in bending stress and the increase in axial stress counteract each other.

(2) With increasing inside flow velocity, the vibration amplitude of the upper riser decreases, the vibration amplitude of the lower part increases slightly, and the displacement of the riser presents a trapezoidal distribution with a narrow top and a wide bottom. The inside flow velocity has little effect on the bending stress in the middle of the riser but has great influence on the bending stress in the lower part. The bending stress in the upper part of the riser increases first and then decreases with the increasing flow velocity, and the bending stress in the middle and lower part of the riser increases with the increase in the flow velocity. The safety of cracked risers decreases with the increase in flow velocity.

(3) The influence of the outside flow velocity on the vibration modes of risers is significant, and the greater the flow velocity, the higher the vibration frequency. The vibration displacement amplitude of the upper end is greatly affected by the flow velocity, while the influence on other parts of the riser is small. The outside flow velocity has great effect on the bending stress, and the greater the flow velocity, the greater the bending stress. With increasing outside flow velocity, the safety of cracks in risers decreases rapidly and significantly.

(4) When other conditions remain unchanged, the vibration displacement amplitude of the middle and lower parts of a riser does not change significantly with an increase in riser length, and the displacement amplitude of the upper part decreases gradually. With an increase in riser length, the bending stress in the riser decreases gradually, but the distribution pattern is more complex. The influence of riser length on crack safety is not significant.

\section{Data Availability}

The data used to support the findings of this study are included within the article.

\section{Conflicts of Interest}

The authors declare that there are no conflicts of interest regarding the publication of this study.

\section{Acknowledgments}

This work has been supported in part by the National Natural Science Foundation of China (Grant nos. 51875489 and 52105125), China Postdoctoral Science Foundation (Grant no. 2021TQ0273), and China Scholarship Council (CSC, File no. 201908510191). 


\section{References}

[1] R. T. Hartlen and I. G. Currie, "Lift-oscillator model of vortexinduced vibration," Journal of the Engineering Mechanics Division, vol. 96, no. 5, pp. 577-591, 1970.

[2] T. Sarpkaya, "Vortex-induced oscillations: a selective review," Journal of Applied Mechanics, vol. 46, no. 2, pp. 241-258, 1979.

[3] H. Y. Guo, Q. Fu, and M. Lou, "Vortex-induced vibrations and fatigue life of marine risers conveying flowing fluid," Engineering Mechanics, vol. 22, no. 4, pp. 220-224, 2005.

[4] J.-j. Gu, C. An, C. Levi, and J. Su, "Prediction of vortex-induced vibration of long flexible cylinders modeled by a coupled nonlinear oscillator: integral transform solution," Journal of Hydrodynamics, vol. 24, no. 6, pp. 888-898, 2012.

[5] D. Yin, E. Passano, and C. M. Larsen, "Improved in-line vortex-induced vibrations prediction for combined in-line and cross-flow vortex-induced vibrations responses," ASME. J. Offshore Mech. Arct. Eng.vol. 140, no. 3, pp. 0318020318028, 2017.

[6] L. Song, S. Fu, J. Cao, L. Ma, and J. Su, "An investigation into the hydrodynamics of a flexible riser undergoing vortex-induced vibration," Journal of Fluids and Structures, vol. 63, pp. 325-350, 2016.

[7] J. Xu, D. Wang, H. Huang, M. Duan, J. Gu, and C. An, "A vortex-induced vibration model for the fatigue analysis of a marine drilling riser," Ships and Offshore Structures, vol. 12, no. 1, pp. 280-287, 2017.

[8] S. Song, Z. Chu, A. Cao et al., "Vortex-parametric coupled vibration characteristics of Deep-Sea top-tension riser," Journal of Harbin University of Engineering, vol. 40, no. 11, pp. 1-6, 2019.

[9] G. Gao, Y. Cui, X. Chou, and Q. Shu, "Parameter influencing analysis of vortex-induced vibration response of deep sea top tensioned riser," Shipbuilding Engineering, vol. 41, no. 2, pp. 101-107, 2019.

[10] S. R. Yu, B. D. Cai, G. Ling, and Z. G. Wang, "Numerical analysis on effect of axial crack on marine riser fatigue life," Journal of Lanzhou University of Technology, vol. 40, no. 1, pp. 59-63, 2014.

[11] M. B. Madjid, A. A. W. Sabah, H. Zahloul, B. Babaziane, M. H. Meliani, and G. Pluvinage, "Finite element analysis of the integrity of an API X65 pipeline with a longitudinal crack repaired with single- and double-bonded composites," Composites Part B, vol. 77, pp. 431-439, 2015.

[12] R. A. Khan and S. Ahmad, "Fatigue reliability assessment of marine risers in deep offshore fields in Indian ocean," ASME 2010 10th Biennial Conference on Engineering Systems Design and Analysis, vol. 2, pp. 1-11, 2010.

[13] X. Shuai, W. Z. Jia, C. F. Zhou, and X. D. Jiao, "The research of crack growth and fatigue life simulation about the girth weld of the deep water riser," Journal of Beijing Institute of Petrochemical Technology, vol. 19, no. 4, pp. 31-34, 2011.

[14] Bs7910, Guide on Methods for Assessing the Acceptability of Flaws in Metallic Structure, British Standard Institution, United Kingdom, 9780580 895647, 2013.

[15] R6, Assessment of the Integrity of Structures Containing Defects, Nuclear Electric Ltd, Glouester, UK, 2001.

[16] Dnv, Offshore Standard-Submarine Pipelines Systems, Det Norske, Veritas, Hovik, Norway, DNV-OS-F101, 2010.

[17] Dnv, Recommended Practice-Fracture Control for Pipeline Installation Methods Introducing Cyclic Plastic Strain, Det Norske, Veritas, Hovik, Norway, DNV-RP-F108, 2012.
[18] M. El-Sayed, A. E. Domiaty, and A. H. I. Mourad, "Fracture assessment of axial crack in steel pipe under internal pressure," Procedia Engineering, vol. 130, pp. 1273-1287, 2015.

[19] M. Li, Z. Cheng, C. Wang, and B. Liu, "Safety assessment for girth weld of X70 pipeline with embedded crack based on BS7910 standard," Journal of Sichuan University of Science \& Engineering, vol. 29, no. 6, pp. 39-43, 2016.

[20] L. S. Tjhen, Y. Zhang, and H. Zhao, "Fracture response of girth-welded pipeline with canoe shape embedded crack subjected to large plastic deformation," Journal of Pressure Vessel Technology, vol. 139, no. 2, 2017.

[21] Y. Zhang, T. L. Seng, and H. Zhao, "Fracture behavior of clad pipeline containing a canoe shape surface crack subjected to large bending moment," Marine Structures, vol. 58, pp. 92108, 2018.

[22] D. K. Yi, S. Idapalapati, Z. M. Xiao, and B. K. Shashi, "Fracture capacity of girth welded pipelines with 3D surface cracks subjected to biaxial loading conditions," International Journal of Pressure Vessels and Piping, vol. 92, pp. 115-126, 2012.

[23] Y. M. Zhang, T. K. Tan, Z. M. Xiao, W. G. Zhang, and M. Z. Ariffin, "Failure assessment on offshore girth welded pipelines due to corrosion defects," Fatigue and Fracture of Engineering Materials and Structures, vol. 39, pp. 453-466, 2016.

[24] M. L. Facchinetti, E. D. Langre, and F. Biolley, "Coupling of structure and wake oscillators in vortex-induced vibrations," Journal of Fluids and Structures, vol. 19, pp. 123-140, 2004.

[25] J. Vandiver, "Drag coefficients of long flexible cylinders," in Proceedings of the Offshore Technology Conference, Houston, Texas, May 1983.

[26] J. Liu, X. Guo, G. Wang et al., "Bi-nonlinear vibration model of tubing string in oil\&gas well and its experimental verification," Applied Mathematical Modelling, vol. 81, pp. 50-69, 2020.

[27] J. Liu, X. Guo, Q. Liu, G. Wang, Y. He, and J. Li, "Vortex induced vibration response characteristics of marine riser considering the in-line and cross-flow coupling effect," Acta Petrolei Sinica, vol. 40, no. 10, pp. 1270-1280, 2019.

[28] C. Y. Deng, Study on the theory and application of leak-beforebreak assessment procedure for the welded pressure pipeline, Ph.D. thesis, University of Tianjin, Tianjin, China, 2006. 